> L'émergence récente d'un nouveau coronavirus, le SARS-CoV-2, responsable de la maladie appelée COVID-19, est un nouvel avertissement du risque pour la santé publique représenté par les zoonoses virales et notamment par les coronavirus. Principalement connus pour leur capacité à infecter les voies respiratoires supérieures et inférieures, les coronavirus peuvent également affecter le système nerveux central et périphérique, comme c'est le cas pour de nombreux virus respiratoires, tels que les virus influenza ou le virus respiratoire syncytial. Les infections du système nerveux sont un problème important de santé publique car elles peuvent provoquer des atteintes dévastatrices allant jusqu'au décès du patient, en particulier lorsqu'elles surviennent chez les personnes fragilisées ou âgées plus sensibles à ce type d'infection. Les connaissances de la physiopathologie des infections par les coronavirus émergents (MERS-CoV, SARS-CoV et SARS-CoV-2) et leurs moyens d'accéder au système nerveux central sont, pour l'heure, très sommaires. Les travaux en cours visent notamment à mieux appréhender les mécanismes associés aux atteintes neurologiques observées. Dans cette revue nous aborderons l'état des connaissances actuelles sur le neurotropisme des coronavirus humains et les mécanismes associés en développant tout particulièrement les dernières données concernant le SARS-CoV-2. <

\section{Les coronavirus humains}

Les coronavirus ( $\mathrm{CoV}$ ) sont des agents pathogènes importants pour les vertébrés en général et l'homme en particulier. Ils peuvent infecter les voies respiratoires, gastro-intestinales, hépatiques mais aussi le système nerveux central (SNC) et périphérique (SNP)

Vignette (Photo (c) Philippe Roingeard et Sébastien Eymieux, Université de Tours, France).

\section{Les atteintes \\ neurologiques liées \\ au SARS-CoV-2 et \\ autres coronavirus \\ humains}

Sara Salinas, Yannick Simonin

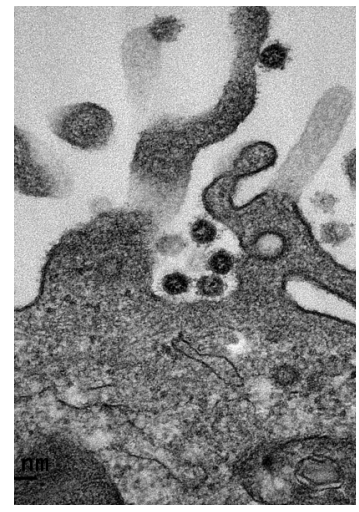

Pathogenèse et contrôle des infections chroniques (PCCI), UMR 1058, université de Montpellier, Inserm, EFS, 60 rue de Navacelles, 34000 Montpellier, France.

sara.salinas@inserm.fr yannick.simonin@umontpellier.fr

de l'homme, du bétail, des oiseaux, des chauves-souris, des rongeurs, ainsi que de nombreux autres animaux sauvages [1]. L'apparition du SARS (severe acute respiratory syndrome)-CoV en 2002/2003 et du MERS (middle East respiratory syndrome)-CoV en 2012 a mis en évidence le potentiel zoonotique des CoV avec une possibilité de transmission initiale entre l'animal et l'homme, puis une transmission d'homme à homme associée à une contagiosité, une morbidité et une mortalité variable. En 2019, l'apparition du SARS-CoV-2, responsable de la maladie COVID-19 (coronavirus disease-19), dans la province du Hubei en République populaire de Chine a confirmé le risque de l'émergence de nouveaux CoV dans la population humaine. Malgré des mesures sanitaires sans précédent, le SARS-CoV-2 a pu rapidement s'étendre sur la totalité du globe, provoquant une pandémie inédite. Le 30 janvier 2020, le directeur général de l'Organisation mondiale de la santé (OMS) a déclaré l'épidémie de SARS-Cov-2 comme une urgence de santé publique de portée internationale. Fin mai 2020, l'OMS avait notifié plus de cinq millions de cas confirmés et près de trois cent cinquante mille décès dans le monde (au 9 juillet 2020, plus de 12 millions de cas confirmés et près de 550000 décès étaient répertoriés par le CSSE - Center for Systems Science and Engineering de l'université américaine John Hopkins [Baltimore, États-Unis]). Tous les continents ont été touchés et plus de 200 pays et territoires ont signalé au moins un cas de COVID-19. L'émergence sporadique et les flambées de nouveaux types de CoV nous rappellent que ces virus sont une grave menace pour la santé mondiale. Les différents CoV présentent une gamme d'hôtes et un tropisme tissulaire variés. Ils appartiennent à l'ordre des Nidovirales, la famille des Coronaviridae et la sous-famille des Coronavirinae. On distingue quatre 
A

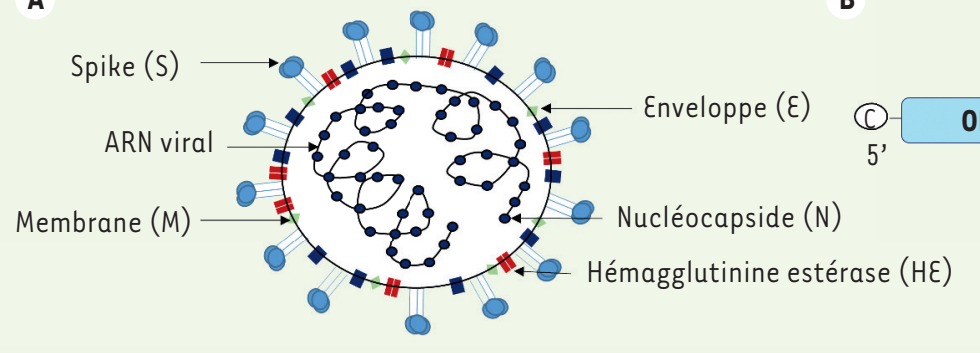

B

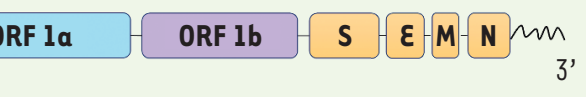

Figure 1. Structure et organisation des coronavirus. A. Vue d'ensemble de la particule virale enveloppée à capside de symétrie hélicoïdale. Taille d'environ 120 nanomètre. B. Représentation simplifiée du génome viral. ARN simple brin de polarité positive d'environ $30 \mathrm{~kb}$. Les deux premiers cadres de lecture ouvert (ORF la et lb) codent notamment les protéines nécessaires au complexe de réplication et les derniers cadres de lecture ouvert les protéines structurales $S, \varepsilon, M$ et $N$. Il existe plusieurs ORF codant des protéines accessoires variables en fonction des coronavirus (non représentés ici) qui ne sont pas essentiels à la réplication in vitro. L'ARN viral possède une coiffe en 5' et une queue Poly $(A)$ en 3 '.

genres de Coronavirinae : les alphacoronavirus, les bêtacoronavirus, les gammacoronavirus et les deltacoronavirus. Les alphacoronavirus et les bêtacoronavirus infectent les mammifères, dont l'homme. Les gammacoronavirus et les deltacoronavirus infectent principalement les oiseaux et les poissons [1]. Sept CoV humains ( $\mathrm{HCoV}$ ) ont été identifiés. Quatre d'entre eux provoquent des atteintes mineures des voies respiratoires supérieures avec de rares complications associées: HCoV-229E (alphacoronavirus), -0C43 (bêtacoronavirus), -NL63 (alphacoronavirus), -HKU1 (bêtacoronavirus). Deux autres, le SARS-CoV et le MERS-CoV, sont des bêtacoronavirus émergents hautement pathogènes pouvant infecter les voies respiratoires inférieures et provoquer un syndrome respiratoire sévère chez l'homme [1]. Le SARS-CoV-2 appartient également aux bêtacoronavirus. II est moins bien décrit que les autres $\mathrm{HCoV}$ en raison de son émergence récente. La séquence de son génome $\rightarrow$ Voir la synthèse de présente une forte homologie avec celle du $\quad$. Sallard et al. page 783 SARS-CoV $(\rightarrow)$ [53], d'où sa dénomination de ce numéro

[2]. Le tableau clinique de l'infection par le SARS-CoV-2 est vaste, allant d'un état asymptomatique à une infection modérée des voies respiratoires supérieures associées à de la fièvre ou de la toux. Différentes atteintes neurologiques ont également été signalées [3]. Les symptômes respiratoires peuvent évoluer vers une forme sévère avec une atteinte des voies respiratoires inférieures pouvant aboutir à un syndrome de détresse respiratoire aiguë (SDRA). L'analyse des bases de données des séquences actuellement connues révèle que tous les coronavirus humains identifiés ont une origine animale: MERS-CoV, SARS-CoV, SARS-CoV-2, HCoV-NL63 et HCoV-229E sont considérés comme provenant de la chauve-souris $[1,4]$; les HCoV OC43 et HKUl seraient issus de rongeurs [1]. Les animaux domestiques peuvent également avoir un rôle important dans la transmission comme hôtes intermédiaires. La transmission à l'homme du MERS-CoV, du SARS-CoV et du SARS-CoV-2 a impliqué un hôte intermédiaire : dromadaire pour le MERS-CoV et civettes pour le SARS-CoV [1] ; l'hôte intermédiaire du SARS-CoV-2 n'est pour l'heure pas clairement identifié même si l'hypothèse du rôle du pangolin ou des civettes a été émise [5].

\section{Structure des coronavirus}

Les CoV sont des virus enveloppés d'environ 120 nanomètres, présentant une capside à symétrie hélicoïdale. Leur génome est un ARN simple brin de polarité positive d'environ 30 kilobases ( $k b$ ) en moyenne (donc parmi les plus grands des virus à $A R N$ ). Ils possèdent une coiffe en 5' et une queue poly (A) en 3' [2] (Figure 1). L'ARN génomique possède au moins 6 cadres de lecture ouverts (open reading frame, ORF). Les premiers ORF (ORFla/b), qui constituent environ les deux tiers du génome, codent pour 16 protéines non structurales permettant notamment la formation du complexe de transcription/réplication. Les ORF situés près de l'extrémité 3' de l'ARN codent au moins pour quatre protéines structurales principales que l'on retrouve chez tous les CoV : Spike (S), la protéine membranaire $(M)$, la protéine d'enveloppe $(\varepsilon)$ et la protéine de nucléocapside (N) (Figure 1). La glycoprotéine S (qui forme une couronne autour du virus, d'où le terme de coronavirus), constituée de deux sous-unités Sl et $\mathbf{S 2}$, est indispensable à l'entrée du virus dans les cellules hôtes. La protéine $\varepsilon$, hautement hydrophobe, est la composante principale de l'enveloppe virale. La protéine $M$ comporte un domaine transmembranaire ainsi qu'une longue partie carboxy-terminale qui peut interagir avec la protéine de nucléocapside N. Elle est principalement impliquée dans l'assemblage des virions ainsi que dans le maintien de la structure virale. La protéine $\mathrm{N}$ possède dans sa partie $\mathrm{N}$-terminale, un domaine de liaison à l'ARN : elle s'associe ainsi à l'ARN viral lors de l'assemblage du virion et permet l'empaquetage du génome à l'intérieur de la capside. Entre ces cadres de lecture, sont localisées des ORF codant des protéines accessoires, dont le nombre et la fonction sont spécifiques de chaque CoV. 


\begin{tabular}{|c|c|}
\hline HCoV (références) & Atteintes neurologiques \\
\hline & Encéphalite \\
\hline & Méningite virale \\
\hline HCoV-229ع [31-32] & Myosite \\
\hline $\mathrm{HCoV}-0 \mathrm{C} 43[29-32 ; 34-36]$ & Encéphalomyélite \\
\hline MERS-CoV [41-43] & Inflammation du tronc cerébral \\
\hline \multirow[t]{6}{*}{ SARS-CoV-2 [45-47; 50-52] } & Syndrome de Guillain-Barré \\
\hline & Syndrome de Miller-Fichser \\
\hline & Syndrome de Guillain-Barré \\
\hline & $\begin{array}{l}\text { Encéphalite hémorragique nécrosante } \\
\text { aiguë }\end{array}$ \\
\hline & Anosmie, dysgueusie \\
\hline & Crise convulsive, conclusion \\
\hline
\end{tabular}

Tableau I. Liste des HCoV et manifestations cliniques neurologiques associées aux infections.

\section{Cycle viral}

L'attachement du virion à la cellule hôte est initié par les interactions du domaine RBD (receptor-binding domain) de la protéine $S$ avec son récepteur, ce qui détermine le spectre d'hôte mais aussi le tropisme tissulaire du virus. Le SARS-CoV cible ainsi les cellules épithéliales bronchiques ciliées et les pneumocytes de type II en se fixant notamment sur l'enzyme de conversion de l'angiotensine 2 (ACદ2) présente à la surface de ces cellules [6]. C'est également le cas du HCoV-NL63 ou du SARS-CoV-2 qui utilisent le même récepteur. Le MERS utilise la dipeptidyl peptidase 4 (DPP4) comme récepteur et infecte les cellules épithéliales bronchiques non ciliées et les pneumocytes de type II [7]. Après l'attachement, une protéase de la cellule hôte clive la protéine $S$, la rendant ainsi active. Selon la protéase utilisée, le virus entre dans la cellule hôte par endocytose (via des vésicules de clathrine et de cavéoline) ou par fusion directe de l'enveloppe virale avec la membrane cellulaire. Dans la cellule hôte, la réplication est assurée par un complexe multi-protéique contenant notamment I'ARN polymérase ARN-dépendante qui assure la réplication du génome. Les virus produits sont ensuite libérés de la cellule hôte via des vésicules sécrétoires.

\section{Le potentiel neuroinvasif des coronavirus}

Bien qu'ayant un tropisme respiratoire, les $\mathrm{HCoV}$ sont également associés à différentes atteintes neurologiques plus ou moins sévères, telles que des encéphalites, des encéphalomyélites, des syndromes de Guillain-Barrél, des anosmies, ou des encéphalopathies aiguës

Maladie auto-immune avec atteinte des nerfs périphériques. nécrosantes $[8,9]$ (Tableau I). Bien que le lien de cause à effet n'ait pas été clairement démontré, de I'ARN de HCoV-OC43 et de HCoV-229દ a également été détecté dans le cerveau de patients souffrant de sclérose en plaques. Dans une cohorte pédiatrique, une étude portant sur les infections par HCoV par détection d'immunoglobulines d'isotype IgM spécifiques du virus dans le plasma de patients souffrant d'encéphalopathies, a révélé que plus de $10 \%$ d'entre eux présentaient des anticorps anti-HCoV, suggérant une infection concomitante qui serait responsable des atteintes neurologiques de type crise convulsive, céphalée et anomalies de la région périventriculaire [10]. Le HCoV0C43, qui a été largement étudié, a été décrit comme pouvant provoquer des neuropathies chez l'homme, en particulier des encéphalites, pouvant dans certains cas être fatales [11-13]. Le virus a également été retrouvé dans le liquide céphalo-rachidien (LCR) d'un enfant malade présentant des lésions de type démyélinisation au niveau du cerveau et de la moelle épinière [11], ainsi que dans une biopsie cérébrale d'un autre enfant souffrant d'une encéphalomyélite [12]. La présence de SARS-CoV a par ailleurs été détectée dans le cerveau de personnes décédées à la suite d'une infection $[14,15]$ et une analyse de cohortes de patients infectés par le MERS-CoV a révélé la présence chez ces patients de symptômes neurologiques sévères, incluant des altérations de conscience, un coma, une ataxie, un déficit moteur focal, des encéphalomyélites et des encéphalites [16-18].

La question de l'impact neurologique de l'infection par le SARS-CoV2 s'est posée assez rapidement au début de la pandémie de COVID-19 [19]. Des atteintes périphériques, telles que les syndromes de Guillain-Barré et de Miller-Fisher ${ }^{2}$, ont en effet été rapportées chez certains patients $[20,21]$ et la présence d'ARN viral a été mise en évidence dans le LCR d'un patient présentant une méningite consécutive à l'infection [22]. Au-delà des différentes atteintes neurologiques que l'on retrouve au cours d'infections par de nombreux virus neurotropes et qui résultent d'une réponse inflammatoire exacerbée, des atteintes plus spécifiques ont été identifiées chez les patients infectés par le SARS-CoV-2. C'est le cas notamment d'hémorragies intracérébrales [3], probablement liées à l'impact du SARS-CoV2 sur la vascularisation. De nombreux cas de confusion, de vertige ou de convulsion ont également été rapportés, sans que le lien avec des atteintes neurologiques directes n'ait été

\footnotetext{
${ }^{2}$ Le syndrome de Miller-Fisher est une variante du syndrome de Guillain-Barré, caractérisée par un manque de coordination des mouvements volontaires (ataxie), une absence de réflexes (aréflexie) et une paralysie des muscles moteurs des yeux
} (ophtalmoplégie). 
démontré [3]. Des atteintes périphériques se traduisant par la perte de l'odorat (anosmie) et du goût (dysgueusie) sont communément observées. Elles pourraient résulter d'un tropisme particulier pour les neurones sensoriels de l'épithélium nasal, voire du bulbe olfactif [3]. Des analyses par IRM (imagerie par résonance magnétique) montrent aussi des atteintes caractéristiques d'encéphalopathie chez les patients [23].

Certaines pathologies associées aux HCoV sont également observées dans des modèles murins d'infection, en particulier chez des souris transgéniques exprimant le récepteur humain ACE2 (hACE2) [24, 25]. Bien qu'imparfaits, notamment au regard des différences de distribution des récepteurs cellulaires entre l'homme et les souris, ces modèles animaux permettent néanmoins une meilleure compréhension de la physiopathologie des infections à HCoV. Comme pour d'autres $\mathrm{HCoV}$, des observations récentes réalisées dans le modèle de souris exprimant hACE2 et infectées par le SARS-CoV2, révèlent, outre un tropisme pulmonaire et une inflammation associée importante, un accès au SNC pouvant préfigurer des troubles neurologiques [26].

\section{Mécanismes d'accès aux systèmes nerveux central et périphérique}

De nombreux agents pathogènes ont été sélectionnés au cours de l'évolution pour leur capacité à accéder au SNC et au SNP. De par sa nature compartimentalisée (il est séparé de la circulation systémique), l'accès au SNC requiert une interaction avec des structures spécialisées, telles que la barrière hématoencéphalique (BHE), constituée en particulier d'une monocouche de cellules endothéliales, ou les synapses périphériques (par exemple les jonctions neuro-musculaires ou sensorielles), qui permettent de réguler l'accès au SNC à des molécules, des cellules ou des agents pathogènes pouvant avoir des effets toxiques. La BHE est une structure essentielle de la protection du SNC. Différents mécanismes permettent néanmoins à certains virus de la franchir : infection directe des cellules endothéliales cérébrales avec libération des virions au niveau du parenchyme; utilisation comme «Cheval de Troie » de cellules du système immunitaire qui peuvent la franchir ; ouverture partielle des jonctions qui résulte d'un environnement inflammatoire au niveau systémique [27]. Le passage ou la perturbation de la $B H \varepsilon$ pourrait ainsi être utilisé par certains CoV, mais peu d'études se sont intéressées à ces mécanismes (Figure 2A). Le MHV-3, un CoV murin, peut induire, par exemple, une augmentation de perméabilité de la BHE [28]. In vitro, il est également capable d'infecter des lignées de cellules endothéliales cérébrales humaines, ce qui suggère la possibilité d'une infection directe par ce virus des cellules endothéliales de la barrière, in vivo [28], qui permettrait sa migration transendothéliale [28]. Un tropisme particulier pour la BHE pourrait aussi résulter de l'expression par les cellules endothéliales cérébrales d'ACE2, le récepteur du virus, ce qui permettrait à celuici d'accéder au SNC [29]. Le tropisme des CoV pour certaines cellules du système immunitaire pourrait également favoriser l'invasion du SNC, par un mécanisme de «Cheval de Troie», comme cela a été montré pour de nombreux virus neurotropes. Dans le modèle de souris trangéniques exprimant hACE2 et infectées par le SARS-CoV2, la présence d'antigènes viraux a ainsi été détectée au niveau des poumons, dans des macrophages [26], dont la capacité à traverser la BHE est bien documentée. Ces macrophages expriment ACE2 et leur infection par les HCoV pourrait donc représenter une voie d'entrée pour le virus dans le SNC (Figure 2A) [30].

Certains virus exploitent également la machinerie de transport axonal pour atteindre le SNC et le SNP [31] (Figure 2B). Ce processus repose sur la polarisation unique des microtubules axonaux et sur l'interaction, complexe et régulée, des moteurs moléculaires et du système endocytique. Faisant suite à l'internalisation d'un agent pathogène et à son transport axonal, la transcytose synaptique permet alors sa dissémination, à l'origine de possibles atteintes du SNC relativement étendues. Pour les virus respiratoires, plusieurs voies d'accès au SNC sont possibles, notamment via l'épithélium nasal, par les neurones récepteurs olfactifs (NRO) [32] ou par le nerf trigéminal [33]. Les NRO jouent un rôle majeur dans le processus d'olfaction et sont particulièrement dynamiques (régénération des cellules et réorganisation). Leur corps cellulaire étant localisé dans l'épithélium olfactif, leur infection directe à ce niveau permet ensuite au virus d'accéder au système de transport axonal antérograde et au bulbe olfactif [32]. L'épithélium nasal pourrait ainsi représenter une cible privilégiée pour les HCoV [34]. La forte expression d'ACE2 par les cellules sustentaculaires de cet épithélium pourrait conférer à ces cellules une forte sensibilité à l'infection et expliquer en partie la perte d'odorat que l'on observe chez certains patients infectés par le SARS-CoV-2 [34]. Contrairement aux cellules sustentaculaires, les NRO expriment peu ACE2 [34]. Le rôle de ce récepteur dans le tropisme neuronal de certains $\mathrm{HCoV}$ et dans les atteintes associées reste donc à déterminer. Une accumulation de virus qui infecterait secondairement les cellules neuronales pourrait cependant avoir pour origine sa réplication locale au sein de cellules non neuronales, par des mécanismes peu dépendants ou indépendants d'ACE2. Cette hypothèse d'invasion indépendante des récepteurs (en anglais, receptor-independent spread) a été suggérée pour certains CoV murins pour lesquels il a été montré que des cellules permissives peuvent transmettre le virus à des cellules moins permissives (n'exprimant pas de récepteur) par transfert de matériel intracellulaire suite à une fusion membranaire, ce qui pourrait expliquer en partie l'infection neuronale après exposition au virus de l'épithélium nasal 
A

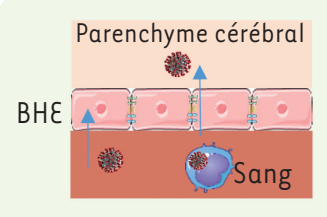

B

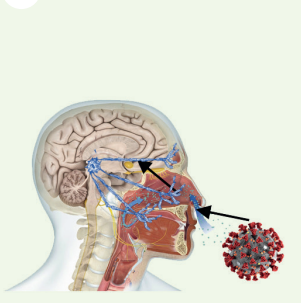

Nerf olfactif?

Nerf trigéminé?
Bulbe olfactif

Nerf olfactif

Épithélium oTfactif

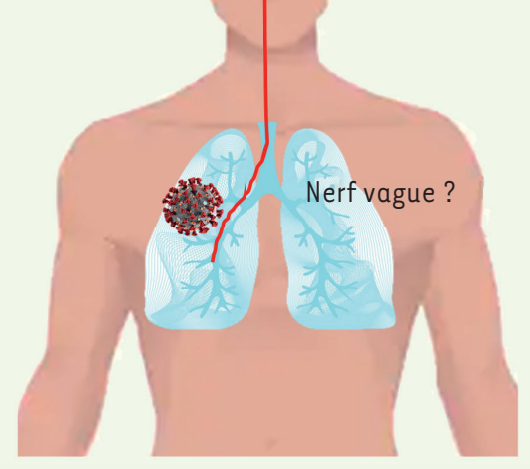

Figure 2. Voies d'accès au système nerveux. Plusieurs mécanismes d'accès ont été décrits pour les HCoV. A. Mécanisme potentiel d'accès au système nerveux central (SNC) par les HCoV via la barrière hémato-encéphalique (BHE) : infection de cellules du système immunitaire type macrophage ou via du virus libre dans le sang. B. Mécanisme potentiel d'accès au SNC par les HCoV via l'épithélium olfactif. Infection de l'épithélium olfactif puis mécanisme de transport axonal via le nerf olfactif jusqu'au bulbe olfactif avant dissémination dans le SNC. Les nerfs vagues (poumons) et trigéminé (fosse nasale) pourraient potentiellement servir de voies d'entrée et/ou de dissémination.

[35]. Les CoV sont aussi capables d'utiliser le transport axonal afin d'entrer dans le SNC et de s'y disséminer. Ainsi, le HCoV-OC43, par exemple, accède au bulbe olfactif après avoir infecté l'épithélium nasal [36]. Les mécanismes de colonisation à partir de l'infection des NRO par le virus puis de son transport vers le bulbe olfactif et vers les autres régions du SNC, via le cortex piriforme, ont ainsi été identifiés [36]. Dans le modèle de souris transgéniques exprimant le récepteur hACE2, I'inoculation intranasale de SARS-CoV provoque une infection importante des poumons mais aussi du cerveau [37]. Le bulbe olfactif est, ici encore, une des premières structures cérébrales dans laquelle une réplication virale est observée [38]. Le virus apparaît ensuite dans des structures possédant des connections de premier ou de deuxième ordre avec le bulbe olfactif, tels que certaines régions du cortex, les ganglions de la base et le mésencéphale, très certainement par un transport axonal et une transcytose synaptique [38]. L'épithélium pulmonaire est fortement innervé. II représente ainsi également une porte d'entrée dans le SNC pour certains virus respiratoires, dont les HCoV. Toutefois, les mécanismes d'infection des neurones au niveau des poumons restent très mal documentés.

\section{Mécanismes de neurovirulence}

La destruction directe par certains virus neurotropes de cellules neuronales, notamment par apoptose, est l'une des principales causes de neurodégénérescence induite lors d'une infection. Un autre mécanisme majeur repose sur l'activation d'une réponse inflammatoire de l'hôte, appelée neuroinflammation. Cette réponse survient principalement au stade aigu de l'invasion du SNC. L'infection virale induit en effet des réponses inflammatoires qui ont pour but un contrôle, voire une inhibition de la réplication virale. Celles-ci peuvent néanmoins s'avérer délétères lorsqu'elles sont excessives. Les cellules cérébrales, dont les macrophages résidents et la microglie, les cellules endothéliales, les neurones et la glie (astrocytes et oligodendrocytes) produisent en effet des cytokines inflammatoires et des chimiokines en réponse à l'invasion, permettant le recrutement, dans les compartiments infectés du SNC, de leucocytes afin de résoudre l'infection. La réponse inflammatoire ainsi induite, si elle n'est pas modulée, peut néanmoins conduire à un « orage cytokinique ». Celui-ci résulte de la production massive et incontrôlée par les cellules présentes de cytokines pro-inflammatoires et anti-inflammatoires, qui seront potentiellement délétères pour le tissu nerveux.

Les mécanismes de neurovirulence associée aux $\mathrm{HCoV}$ sont peu décrits, notamment pour les CoV émergents, en particulier pour le SARS-CoV-2. Certaines études, 
impliquant notamment le $\mathrm{HCOV}-0 \mathrm{C} 43$, ont identifié la protéine virale $\mathrm{S}$ comme étant l'un des principaux déterminants de la pathogenèse du virus. Spike est une protéine transmembranaire de type I, fortement $\mathrm{N}$-glycosylée, de 180 à 200 kDa. Elle s'assemble en homotrimères à la surface de la particule virale [39]. Elle joue un double rôle lors de l'entrée du virus. Elle permet la liaison du virus à la cellule, via son récepteur. Elle est également impliquée dans la fusion de l'enveloppe virale avec la membrane de la cellule cible. Spike est ainsi déterminante pour le tropisme cellulaire et donc pour la pathogénicité du virus, étant associée à de nombreux effets cytotoxiques qui conduisent à la dégénérescence des cellules infectées par différents CoV $[40,41](\rightarrow)$.

$(\rightarrow)$ Voir la Nouvelle de P.J. Talbot et al., $m / s n^{\circ} 8-9$, août-septembre 2016, page 696

Spike participe également à la neurovirulence du virus. Le HCoV-OC43 induit en effet, dans un modèle murin d'infection, l'infiltration de cellules immunitaires et la production de cytokines dans le SNC [24]. Cette réponse inflammatoire est exacerbée par des variants du virus dont la glycoprotéine S a été mutée, augmentant notamment l'excitotoxicité du glutamate, ce qui pourrait expliquer la dégénérescence neuronale du SNC et la demyélination de la moelle épinière observées [42]. Le HCoV-OC43 peut également infecter les neurones dans différentes régions du cerveau et induire leur mort, notamment par un processus d'apoptose induit par spike, indépendant des caspases [43]. Ces observations sont à mettre en relation avec celles faites avec les coronavirus murins MHV-A59 et MHVJHM pour lesquels il a été montré que spike joue un rôle important dans la capacité d'invasion neuronale des virus, avec en particulier leur action sur la démyélinisation des neurones [44]. Pour les HCoV émergents, comme le SARS-CoV, le SARS-CoV-2 ou le MERS-CoV, malgré son implication dans leur infectivité, le rôle de la protéine $S$ dans la neurovirulence n'est pas élucidé $[1,45,46]$. Pour le MERS-CoV, l'utilisation de souris transgéniques exprimant le récepteur humain du virus (hDPP4) a néanmoins révélé la présence, après infection, de nécrose neuronale dans le cortex cérébral, notamment au niveau des neurones hippocampaux. Dans ce modèle, le virus a été identifié non seulement dans le corps cellulaire des neurones, mais aussi au niveau des dendrites et des cellules de la microglie [47]. Pour le SARS-CoV, le tropisme du virus pour le SNC et les lésions neuronales associées à l'infection ont été caractérisés dans des études expérimentales et cliniques, montrant notamment que les cellules neuronales humaines exprimant ACE2 peuvent être infectées [14, 15, 48, 49]. L'étude des mécanismes impliqués dans la neurodégénéresence induite par le virus est cependant complexe. En effet, bien que le SARS-CoV se réplique chez de nombreuses espèces, notamment la souris ou les primates non humains, il ne provoque pas à l'origine, de façon reproductible chez ces animaux, une maladie dont la gravité serait équivalente au SARS observé chez l'homme. Les souris transgéniques exprimant hACE2 sont néanmoins sensibles à l'infection et la présence de virus dans le cerveau des animaux infectés est détectée [50]. Dans ce modèle, malgré une faible infiltration cellulaire au niveau du cerveau, une forte expression de différentes cytokines pro-inflammatoires, dont I'IL(interleukine)-6, est observée, suggérant un possible déclenchement d'un orage cytokinique induit par l'infection. Chez les souris infectées, de nombreuses zones du cerveau (comme le gyrus denté de l'hippocampe ou le cervelet) contiennent du virus, avec une perte neuronale massive [38]. Le bulbe olfactif est également infecté, ainsi que le complexe vagal dorsal, la partie caudale du tronc cérébral, impliquée dans la régulation centrale des fonctions viscérales et critique pour la fonction respiratoire. Une expression importante de la chimiokine CXCL9 ( $C-X-C$ motif chemokine ligand 9) a été observée dans le cerveau d'un patient infecté par le SARS-CoV. Elle pourrait être impliquée dans les dommages immunopathologiques identifiés dans le cerveau de patients infectés [15]. La communauté de récepteur entre celui du SARS-CoV et celui du SARS-CoV-2 (ACE2) permet d'envisager que les mécanismes de neurovirulence utilisés par les deux virus soient partiellement similaires. Des études supplémentaires restent néanmoins nécessaires. Certaines études ont montré que les protéines $\varepsilon$ et $\mathrm{H} \varepsilon$, ou NS2 et NS5 du HCoV-OC43 étaient également des facteurs importants pour la neurovirulence du virus. Leurs rôles restent cependant à déterminer pour les autres HCoV à tropisme neurologique [51].

\section{Diagnostic et traitement}

Dans la plupart des cas, le diagnostic d'infection par CoV n'est pas réalisé, la maladie étant généralement bégnine et ne touchant que les voies respiratoires hautes. Cette notion a néanmoins été ré-évaluée avec l'émergence des nouveaux $\mathrm{CoV}$, beaucoup plus délétères pour l'homme. En cas d'épidémie, les tests diagnostiques sont principalement effectués dans des laboratoires de santé publique. Ils consistent le plus souvent en un prélèvement naso-pharyngé réalisé par écouvillonnage et détection du matériel génétique viral dans le prélèvement par RT-PCR (reverse transcriptase polymerase chain reaction). Le diagnostic biologique des atteintes du SNC associées à des infections virales a pour but de déterminer l'étiologie permettant d'expliquer les symptômes que le neurologue identifie. L'électroencéphalogramme et l'imagerie médicale (scanner, IRM) peuvent révéler des anomalies caractéristiques, principalement au niveau des lobes temporaux du cerveau. Pour la maladie COVID-19, les images d'IRM cérébrale peuvent notamment révéler des lésions hémorragiques au niveau des thalamus bilatéraux, des lobes temporaux médians ou des régions sous-insulaires. Dans certains cas, les virus impliqués peuvent également être détectés par PCR dans le LCR.

À l'heure actuelle, il n'existe pas de traitement antiviral spécifique contre les CoV : les principaux sont des traitements de soutien, y compris pour les atteintes neurologiques. De nombreuses molécules ciblant notamment les nucléosides, les nucléotides, les acides nucléiques viraux et les enzymes/protéines impliqués dans la réplication et 
la transcription des CoV sont des stratégies prometteuses pour traiter ces infections [52]. La glycoprotéine $S$ est également une cible potentielle importante du fait de son rôle dans l'interaction entre le virus et son récepteur cellulaire, mais aussi du fait de son implication dans la neurovirulence. La recherche de traitements efficaces s'est considérablement accélérée avec la pandémie récente de SARS-CoV-2, et des centaines de molécules sont actuellement en cours de tests à des degrés variables d'avancement.

\section{Conclusion et perspectives}

Le début $d u x x l^{e}$ siècle a été marqué par de multiples émergences et réémergences de maladies virales, notamment de nouveaux CoV, tels que le MERS-CoV, le SARS-CoV et, plus récemment, le SARS-CoV-2. Les changements climatiques et écologiques, ainsi que les interactions entre humains et animaux sont autant de facteurs favorisant ces émergences. La pandémie de COVID-19 souligne les risques sanitaires majeurs que ces virus trop peu caractérisés peuvent engendrer. Bien qu'étant des virus respiratoires, de plus en plus de données soulignent que ces HCoV peuvent également être à l'origine de lésions touchant le système nerveux. Pourtant, les connaissances acquises ne permettent pas encore d'appréhender l'importance de ces atteintes neurologiques sur l'évolution de la maladie. De futures recherches devraient s'orienter sur la compréhension des mécanismes permettant aux HCoV émergeants d'accéder au SNC et sur la caractérisation des déterminants de leur neurovirulence afin, notamment, d'élaborer de futures solutions thérapeutiques ciblées. $\diamond$

\section{SUMMARY}

Neurological damage linked to coronaviruses: SARS-CoV-2 and other human coronaviruses

The recent emergence of a new coronavirus, SARS-CoV-2, responsible for COVID-19, is a new warning of the risk to public health represented by viral zoonoses and in particular by coronaviruses. Mainly described as being able to infect the upper and lower respiratory tract, coronaviruses can also infect the central and peripheral nervous systems as many other respiratory viruses, such as influenza or respiratory syncytial virus. Viral infections of the nervous system are a major public health concern as they can cause devastating illnesses up to death, especially when they occur in the elderly, who are more susceptible to these infections. Knowledge concerning the pathophysiology of recently emerging coronaviruses (MERS-CoV, SARS-CoV and SARSCOV-2) and how they reach the central nervous system are very sketchy and the work in progress aims in particular to better understand their biology and the mechanisms associated with neurological damage. In this review we will discuss the current state of knowledge on the neurotropism of human coronaviruses and the associated mechanisms by developing in particular the latest data concerning SARS-CoV-2. $\diamond$

\section{LIENS D'INTÉRÊT}

Les auteurs déclarent n'avoir aucun lien d'intérêt concernant les données publiées dans cet article.

\section{RÉFÉRENCES}

1. Cui J, Li F, Shi ZL. Origin and evolution of pathogenic coronaviruses. Nat Rev Microbiol $2019 ; 17$ : 181-92.

2. Chen $Y$, Liu Q, Guo D. Emerging coronaviruses: genome structure, replication, and pathogenesis. J Med Virol 2020 ; 92 : 418-23.

3. Mao L, Jin H, Wang M, et al. Neurologic manifestations of hospitalized patients with coronavirus disease 2019 in Wuhan, China. JAMA Neurol 2020 Apr 10;e201127. doi: 10.1001/jamaneurol.2020.1127.

4. Zhou P, Yang X Lou, Wang XG, et al. A pneumonia outbreak associated with a new coronavirus of probable bat origin. Nature $2020 ; 579: 270-3$.

5. Ye ZW, Yuan S, Yuen KS, et al. Zoonotic origins of human coronaviruses. Int J Biol Sci $2020 ; 2020$ : 1686-97.

6. Ge XYY, Li JLL, Yang XL, et al. Isolation and characterization of a bat SARSlike coronavirus that uses the ACE2 receptor. Nature 2013 ; $503: 535-8$.

7. Raj VS, Mou H, Smits SL, et al. Dipeptidyl peptidase 4 is a functional receptor for the emerging human coronavirus-EMC. Nature 2013 ; 495 : 2514.

8. Desforges M, Coupanec A Le, Stodola JK, et al. Human coronaviruses: viral and cellular factors involved in neuroinvasiveness and neuropathogenesis. Virus Res 2014 ; 194 : 145-58.

9. Nath A. Neurologic complications of coronavirus infections. Neurology $2020 ; 94: 809-10$.

10. Li Y, Li H, Fan R, et al. Coronavirus infections in the central nervous system and respiratory tract show distinct features in hospitalized children. Intervirology $2017 ; 59: 163-9$.

11. Ann Yeh $\varepsilon$, Collins A, Cohen ME, et al. Detection of coronavirus in the central nervous system of a child with acute disseminated encephalomyelitis. Pediatrics 2004 ; 113 : e73-6.

12. Morfopoulou S, Brown JR, Davies $\varepsilon G$, et al. Human coronavirus $0 C 43$ associated with fatal encephalitis. N Engl J Med 2016 ; $375: 497-8$.

13. A N, N E, J A, et al. Fatal encephalitis associated with coronavirus $0 C 43$ in an immunocompromised child. Infect Dis $2020 ; 52$.

14. Gu J, Gong $\varepsilon$, Zhang B, et al. Multiple organ infection and the pathogenesis of SARS. J Exp Med $2005 ; 202:$ 415-24.

15. Xu J, Zhong S, Liu J, et al. Detection of severe acute respiratory syndrome coronavirus in the brain: potential role of the chemokine Mig in pathogenesis. Clin Infect Dis 2005 ; 41 : 1089-96.

16. Yuan J, Yang S, Wang S, et al. Mild encephalitis/encephalopathy with reversible splenial lesion (MERS) in adults-a case report and literature review. BMC Neurol $2017 ; 17: 103$.

17. Algahtani H, Subahi A, Shirah B. Neurological complications of Middle East respiratory syndrome coronavirus: a report of two cases and review of the literature. Case Rep Neurol Med 2016; 2016 : 3502683.

18. YM A, A H, J H, et al. Severe neurologic syndrome associated with Middle East respiratory syndrome corona virus (MERS-CoV). Infection 2015; 43.

19. Li YC, Bai WZ, Hashikawa T. The neuroinvasive potential of SARS-CoV2 may play a role in the respiratory failure of COVID-19 patients. J Med Virol 2020 ; 10.1002/jmv.25728. doi: 10.1002/jmv.25728.

20. Sedaghat Z, Karimi N. Guillain Barre syndrome associated with COVID-19 infection: a case report. J. Clin. Neurosci 2020.

21. Gutiérrez-Ortiz C, Méndez A, Rodrigo-Rey S, et al. Miller Fisher syndrome and polyneuritis cranialis in COVID-19. Neurology 2020 ; 10.1212/ WNL.0000000000009619. doi: 10.1212/WNL.0000000000009619.

22. Moriguchi T, Harii N, Goto J, et al. A first case of meningitis/encephalitis associated with SARS-coronavirus-2. Int J Infect Dis 2020 ; $94: 55-8$.

23. Helms J, Kremer S, Merdji H, et al. Neurologic features in severe SARS-CoV-2 infection. N Engl J Med 2020 ; NEJMc2008597. doi: 10.1056/NEJMc2008597.

24. Jacomy H, St-Jean JR, Brison $\varepsilon$, et al. Mutations in the spike glycoprotein of human coronavirus $\mathrm{OC} 43$ modulate disease in BALB/c mice from encephalitis to flaccid paralysis and demyelination. J Neurovirol $2010 ; 16: 279-93$.

25. Carmo S Do, Jacomy H, Talbot PJ, et al. Neuroprotective effect of apolipoprotein D against human coronavirus 0 C43-induced encephalitis in mice.J Neurosci $2008 ; 28: 10330-8$.

26. Bao L, Deng W, Huang B, et al. The pathogenicity of SARS-CoV-2 in hACE2 transgenic mice. Nature 2020 ; doi: 10.1038/s41586-020-2312-y

27. Miner JJ, Diamond MS. Mechanisms of restriction of viral neuroinvasion at the blood-brain barrier. Curr Opin Immunol $2016 ; 38: 18-23$.

28. Bleau C, Filliol A, Samson M, et al. Brain invasion by mouse hepatitis virus depends on impairment of tight junctions and beta interferon production in brain microvascular endothelial cells. J Virol 2015 ; 89 : 9896-908. 


\section{RÉFÉRENCES}

29. Harmer D, Gilbert M, Borman R, et al. Quantitative mRNA expression profiling of ACE 2, a novel homologue of angiotensin converting enzyme. FEBS Lett $2002 ; 532: 107-10$.

30. Magrone T, Magrone M, Jirillo $\varepsilon$. Focus on receptors for coronaviruses with special reference to angiotensin-converting enzyme 2 as a potential drug target: a perspective. Endocr Metab Immune Disord Drug Targets 2020 ; 20 : doi: 10.2174/1871530320666200427112902.

31. Salinas $S$, Schiavo G, Kremer $\varepsilon$ J. A hitchhiker's guide to the nervous system: the complex journey of viruses and toxins. Nat Re Microbiol $2010 ; 8: 645-55$

32. Kalinke U, Bechmann I, Detje CN. Host strategies against virus entry via the olfactory system. Virulence $2011 ; 2$

33. Menendez CM, Carr DJJ. Defining nervous system susceptibility during acute and latent herpes simplex virus-1 infection. J Neuroimmunol $2017 ; 308: 43-9$.

34. Bilinska K, Jakubowska P, Bartheld CS VON, et al. Expression of the SARS-CoV-2 entry proteins, ACE2 and TMPRSS2, in cells of the olfactory epithelium: identification of cell types and trends with age. ACS Chem Neurosci 2020 ; acschemneuro.0c00210. doi: 10.1021/acschemneuro.0c00210.

35. Hoffmann M, Kleine-Weber H, Schroeder S, et al. SARS-CoV-2 cell entry depends on ACE2 and TMPRSS2 and Is blocked by a clinically proven protease inhibitor. Cell $2020 ; 181: 271-280 . e 8$.

36. Dubé M, Coupanec A Le, Wong AHM, et al. Axonal transport enables neuron-to-neuron propagation of human coronavirus OC43. J Virol $2018 ; 92$.

37. Tseng CTK, Huang C, Newman P, et al. Severe acute respiratory syndrome coronavirus infection of mice transgenic for the human angiotensin-converting enzyme 2 virus receptor. J Virol $2007 ; 81$ : 1162-73.

38. Netland J, Meyerholz DK, Moore S, et al. Severe acute respiratory syndrome coronavirus infection causes neuronal death in the absence of encephalitis in mice transgenic for human ACE2.J Virol $2008 ; 82: 7264-75$.

39. Delmas B, Laude H. Assembly of coronavirus spike protein into trimers and its role in epitope expression. J Virol $1990 ; 64: 5367-75$

40. Millet JK, Whittaker GR. Host cell proteases: critical determinants of coronavirus tropism and pathogenesis. Virus Res $2015 ; 202: 120$-34.

41. Talbot PJ, Desforges M, Dubé $M$, et al. Coronavirus respiratoires humains neurotropes : une relation ambiguë entre neurovirulence et clivage protéique. Med Sci (Paris) 2016 ; 32 : 696-9.

42. Brison $\varepsilon$, Jacomy $H$, Desforges $M$, et al. Glutamate excitotoxicity is involved in the induction of paralysis in mice after infection by a human coronavirus with a single point mutation in its spike protein. J Virol 2011; $85: 12464-73$.
43. Favreau DJ, Meessen-Pinard M, Desforges M, et al. Human coronavirusiinduced neuronal programmed cell death is cyclophilin $D$ dependent and potentially caspase dispensable. J Virol 2012 ; 86 : 81-93.

44. Bender SJ, Weiss SR. Pathogenesis of murine coronavirus in the central nervous system. J Neuroimmune Pharmacol $2010 ; 5$ : 336-54.

45. Falzarano D, Wit $\varepsilon$ de, Feldmann F, et al. Infection with MERS-CoV causes lethal pneumonia in the common marmoset. PLoS Pathog $2014 ; 10$.

46. Reinke LM, Spiegel M, Plegge T, et al. Different residues in the SARS-CoV spike protein determine cleavage and activation by the host cell protease TMPRSS2. PLoS One 2017 ; 12.

47. Zhao $\mathrm{G}$, Jiang $Y$, Qiu $H$, et al. Multi-organ damage in human dipeptidyl peptidase 4 transgenic mice infected with Middle East respiratory syndrome-coronavirus. PLoS One $2015 ; 10$.

48. Lau KK, Yu WC, Chu CM, et al. Possible central nervous system infection by SARS coronavirus. Emerg Infect Dis $2004 ; 10: 342-4$.

49. Yamashita M, Yamate M, Li GM, et al. Susceptibility of human and rat neura cell lines to infection by SARS-coronavirus. Biochem Biophys Res Commun $2005 ; 334: 79-85$

50. McCray PB, Pewe L, Wohlford-Lenane C, et al. Lethal infection of K18-hACE2 mice infected with severe acute respiratory syndrome coronavirus. J Virol $2007 ; 81: 813-21$

51. Desforges M, Coupanec A Le, Dubeau P, et al. Human coronaviruses and other respiratory viruses: underestimated opportunistic pathogens of the central nervous system? Viruses $2019 ; 12$

52. Zumla A, Chan JFW, Azhar El, et al. Coronaviruses-drug discovery and therapeutic options. Nat Rev Drug Discov 2016 ; 15 : 327-47.

53. Sallard $\varepsilon$, Halloy J, Casane D, et al. Retrouver les origines du SARS-COV-2 dans les phylogénies de coronavirus. Med Sci (Paris) 2020 ; 36 : 783-96

\section{TIRÉS À PART}

Y. Simonin

P ossédées du Malin au Moyen-Âge, les sorcières hystériques sont vouées au bûcher. Enfermées au XVII ${ }^{\circledR}$ siècle, maltraitées, elles rejoignent la Cour des Miracles de l'Hospice de la Vieillesse-Femmes à la Salpêtrière... Jusqu'à ce que le Dr JeanMartin Charcot (1825-1893) mène le combat qui transforme l'ancien hospice en hôpital : l'École de la Salpêtrière de

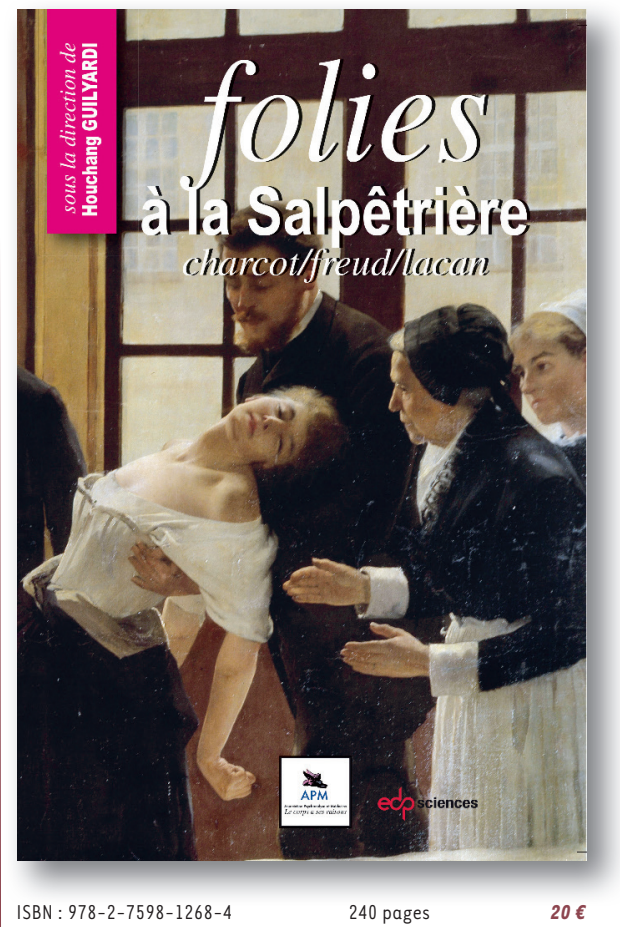
Paris est née, qui devient lieu de recherche, d'enseignement et de soins, de renommée internationale.

Jean Martin Charcot n'a pas bonne presse, et pourtant... Hystérie et folie traversent les siècles, prenant les formes de «l'air du temps».

De l'utérus migrateur d'Hippocrate aux recherches neurologiques de Charcot. Du désir inconscient avec Freud à la jouissance du parlêtre chez Lacan... C'est à cette traversée historique et conceptuelle que nous convie cet ouvrage.

À retourner à EDP Sciences, 17 avenue du Hoggar, 91944 Les Ulis Cedex, France

Tél. : 0149856069 - Fax : 0149850345 - $\varepsilon$-mail : francois.flori@edpsciences.org

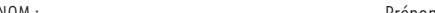

Adress :

Code postal : .............................................. Ville :

Pays :

Fonction :

souhaite recevoir

Folies à la Salpêtrière : $20 €+3 €$ de port $=23 € \pi T$

en ................. exemplaire, soit un total de

$\neg$ Par chèque, à l'ordre de EDP Sciences

$\square$ Par carte bancaire : $\square$ Visa $\square$ Eurocard/Mastercard

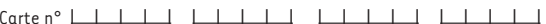

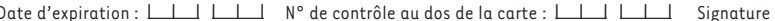

